\title{
Validation of a prediction model for avoiding post-chemotherapy retroperitoneal lymphadenectomy in patients with metastatic non- seminomatous germ cell cancer
}

\author{
Nahid Punjani, MD;' Nicholas Power, MD, FRCSC;' James J. Vanhie, BSC,; Eric Winquist, MD, MSc, FRCPC, FACP2 \\ 'Division of Urology, Western University and London Health Sciences Centre, London, ON, Canada; '2Division of Medical Oncology, Western University and London Health Sciences Centre, London, ON, Canada
}

Cite as: Can Urol Assoc J 2016;10(7-8):260-3. http://dx.doi.org/10.5489/cuai.3558

\section{Abstract}

Introduction: Post-chemotherapy residual masses (PCRMs) may contain persistent cancer or teratoma in more than $50 \%$ of patients with metastatic non-seminomatous germ cell tumours (mNSGCTs). Retroperitoneal lymph node dissection (RPLND) is curative, but controversy exists about selection criteria for surgery. A validated prediction model by Vergouwe et al (2007) based on over 1000 patients was evaluated at our centre.

Methods: mNSGCT patients treated with RPLND for PCRMs were identified from an electronic database. Typographical errors in the model were identified and corrected using their 2003 publication, but retaining the 2007 coefficients. Six clinical variables were included in the model and the calculated probability of benign tissue was compared with pathology. "Benign tissue only" was considered a positive test outcome in patients with a predicted probability of "benign tissue only" greater than $70 \%$.

Results: Fifty-two (52) mNSGCT patients between 1980 and 2014 were evaluable. Median age was 32 years (range 17-52) and International Germ Cell Consensus Classification (IGCCC) prognostic stages were: good $46.2 \%$, intermediate $32.7 \%$, and poor $21.2 \%$. Most patients received bleomycin/etoposide/cisplatin (BEP) chemotherapy and full bilateral RPLND. Pathology showed residual cancer or teratoma in 31 patients (59.6\%) and benign findings in 21 patients $(40.6 \%)$. Positive and negative predictive values and accuracy were $100 \%, 69 \%$, and $73 \%$, respectively.

Conclusions: "Benign tissue only" was found in $100 \%$ of patients in whom this was predicted using our pre-determined criteria. This study involved a limited number of patients, but confirms the potential value of the Vergouwe et al model. Routine use of this prediction model in clinical practice should be tested for mNSGCT patients with PCRMs.

\section{Introduction}

Testicular cancer affects approximately 8400 men in the U.S. each year and constitutes the most common solid tumour in men 15-34 years of age. ${ }^{1}$ Of these, the majority are germ cell tumours, with almost half classified as nonseminomatous germ cell tumours (NSGCTs). ${ }^{2,3}$ Management pathways include a combination of surveillance, chemotherapy, and/or surgery. Metastatic NGSCTs are usually treated with first-line cisplatin chemotherapy, ${ }^{4}$ followed by post-chemotherapy retroperitoneal lymph node dissection (RPLND) for patients with residual masses on computed tomography $(\mathrm{CT})$. $^{5-7}$

All surgical interventions, including RPLND, have associated morbidity and mortality, as well as financial and institutional implications. ${ }^{8}$ Currently, RPLND is recommended in all post-chemotherapy NSGCT patients with a residual mass $1 \mathrm{~cm}$ or greater in size. ${ }^{7}$ There have been efforts to develop prediction tools to avoid surgery in the $40 \%$ of patients whose residual masses are benign, containing only necrotic debris or fibrosis. Several studies have worked to externally validate these models, which have shown significant discrimination, calibration, and clinical utility. ${ }^{9}$ Vergouwe and colleagues published a final version of a nomogram to predict the presence of benign masses that used six clinical parameters, including pre-chemotherapy tumour markers, the presence of teratomatous elements, and both mass shrinkage and residual mass size on postchemotherapy CT scans. ${ }^{4,8}$ The data for the model included over 1000 patients from large academic hospitals, including the Academic Hospital Groningen, Norweigan Radium Hospital and Memorial Sloan Kettering Cancer Cancer, Indiana University, Klinikum Grosshadern München, and the European Organiziation for Research and Treatment of Cancer/Medical Research Council. The criteria for RPLND were at the discretion of each local site, as some centres performed RPLND on all patients and others used a size cutoff. ${ }^{4,8}$ The goal of our study was to review, apply, and externally validate the nomogram developed and reported by Vergouwe et al. ${ }^{10}$ 


\section{Methods}

A comprehensive electronic database, which included all men treated with first-line cisplatin-based chemotherapy for disseminated germ cell tumours at London Health Sciences Centre between January 1980 and December 2014, was retrospectively reviewed. Men subsequently treated with RPLND for a residual retroperitoneal mass greater than 1 $\mathrm{cm}$ following first-line chemotherapy were identified. Data extraction included the six factors applied in the nomogram model: pre-chemotherapy alpha fetoprotein (AFP), human chorionic gonadotropin (HCG) and lactate dehydrogenase (LDH) levels, presence or absence of teratoma elements in the primary tumour, post-chemotherapy residual mass size on CT scan, and change in mass with chemotherapy treatment. Patients were excluded if there was insufficient data for any required parameter or without available final pathology. To reiterate, the Vergouwe model data includes over 1000 patients from many specialized care centres. ${ }^{10}$

As we tested the Vergouwe nomogram, provided in the 2007 publication, we noticed that we could not replicate the example provided in the text and also encountered discordance between results generated by the nomogram and formula when both were applied to the same patients. On careful review of four publications developing and validating a predictive model based on these parameters, we identified three typographical errors in the 2007 published report. $4,8,9,11$ The errors included, "The change in mass size was associated with a lower probability," which was incorrect. There was numerical discordance with a described example of mass size reduction in the nomogram and accompanying text, and the probability of benign disease was described as a positive rather than negative exponent (Fig. 1).

To address these errors, we used the formula described in the 2003 paper with coefficients from the 2007 publication, which had validated this formula with a larger sample size (Fig. 2). ${ }^{9,11}$ The formula provided a prediction of the probability of "benign tissue only" at RPLND. The predicted probabilities were dichotomized using a cutoff of greater than $70 \%$, as suggested by the authors, and were then compared to final pathology. Final pathology was grouped as positive or benign, with positive including any elements of either germ cell cancer or teratoma. ${ }^{10}$

Positive and negative predictive values and accuracy were calculated according to standard definitions with 95\%

\begin{tabular}{|ll|}
\hline 1 & $\begin{array}{l}\text { In the model, "change in mass size" was associated with a } \\
\text { lower probability and this was incorrect }{ }^{10}\end{array}$ \\
\hline 2 & $\begin{array}{l}\text { The example using the nomogram described in the paper } \\
\text { had a numerical discordance with the accompanying text }\end{array}$ \\
\hline 3 & $\begin{array}{l}\text { The overall probability of benign disease in the nomogram } \\
\text { formula was described as a positive rather than negative }^{\text {exponent }}{ }^{10}\end{array}$ \\
\hline
\end{tabular}

Fig. 1. Specific errors. confidence intervals (Cls) on proportions estimated using continuity correction. ${ }^{12,13}$ The study was approved by the Western University research ethics board.

\section{Results}

Between 1980 and 2014, 414 men were treated at London Health Sciences Centre with chemotherapy for testicular cancer. Of these 414 men, 90 subsequently underwent RPLND for residual mass following first-line chemotherapy. Of these 90 men, 38 were excluded due to incomplete data, leaving 52 men eligible for inclusion in the study. No other exclusions were applied.

The 52 men studied were of median age 32 years (range 17-52) (Table 1). Distribution by IGCCC prognostic stages was: good $(46.2 \%)$, intermediate $(32.7 \%)$, and poor $(21.2 \%)$. All but three patients were treated with bleomycin/etoposide/cisplatin (BEP) chemotherapy and all had full bilateral RPLND with or without nerve-sparing.

Final pathology at RPLND and the predicted benign percentage based on the corrected Vergouwe model were compared. Cancer and/or teratoma were found in 31 (59.6\%) patients and $21(40.4 \%)$ had only benign findings. Positive and negative predictive values and accuracy were $100 \%$, $69 \%$, and $73 \%$, respectively. Specificity and sensitivity were $100 \%$ and $33 \%$, respectively (Table 2 ). At a cutoff of $70 \%$, the Vergouwe model predicted "benign only" findings in seven patients and all seven of these patients were found to have pathologically benign disease.

Based on a corrected version of Vergouwe et al's 2007 prediction formula, ${ }^{10,11}$ we have confirmed their model for easy calculation in the clinical environment, as seen in Fig. 3. The three dichotomous variables are listed first, followed by the three continuous variables. A total sum is calculated and the corresponding predicted probability of benign tissue is evident using the bottom scale.

\footnotetext{
Linear predictor $=-1.20+1.13^{*}$ (teratoma-negative $)+$ $1.11 *($ AFPnormal $)+0.72 *($ HCGnormal $)+0.82 *(\ln [\text { LDHst }])^{\pi}-$ $\left.0.27^{*}(\text { sqrt [postsize }]^{\dagger}\right)+0.14^{*}(\text { reduction })^{\ddagger}$
}

The exact probability of benign histology is calculated with the formula:

Probability $=1 /\left(1+\mathrm{e}^{\text {-linear predictor })}\right.$

This probability is approximated by the sum score from nomogram.

"LDH upper limit of normal was adjusted based on laboratory values; ${ }^{+}$in millimeters; ${ }^{*}$ change in tumour size by $10 \%$ increments, i.e., ([presize-postsize]/presize) x 10 .

Fig. 2. Corrected prediction formula. 


\begin{tabular}{lc}
\hline Table 1. Patient characteristics & \\
\hline Characteristic & Patients \\
\hline Demographics & $32(17-52)$ \\
$\quad$ Median age (range), years & \\
Systemic chemotherapy, $n(\%)$ & $49(94)$ \\
BEP & $3(6)$ \\
Other & \\
IGCCC prognostic stages, $n(\%)$ & $24(46)$ \\
Good & $17(33)$ \\
Intermediate & $11(21)$ \\
Poor & \\
\hline BEP: bleomycin, etoposide, cisplatin ; IGCCC: International Germ Cell Consensus \\
Classification.
\end{tabular}

\section{Discussion}

Metastatic NSGCT is managed with cisplatin-based chemotherapy and frequently requires post-chemotherapy RPLND to remove residual retroperitoneal masses. ${ }^{14}$ This surgery has risks and may have long-term effects on patients' health, and is unnecessary in the $40 \%$ of cases with only benign pathological findings. ${ }^{8}$ Several approaches to more precisely applying RPLND in this setting have been suggested, includ-

\section{Table 2. Summary statistics}

\begin{tabular}{lc} 
Sensitivity & $33 \%(95 \% \mathrm{Cl} 15-57)$ \\
Specificity & $100 \%(95 \% \mathrm{Cl} 86-100)$ \\
Positive predictive value & $100 \%(95 \% \mathrm{Cl} 56-100)$ \\
Negative predictive value & $69 \%(95 \% \mathrm{Cl} 53-81)$ \\
Accuracy & $73 \%(95 \% \mathrm{Cl} 58-84)$ \\
\hline Cl: confidence interval.
\end{tabular}

ing size parameters of the retroperitoneal mass. ${ }^{7}$ Clinicians are often limited to imaging to determine if the residual mass is indeed cancer. ${ }^{8}$ Furthermore, an optimal cutoff value has not been reported. ${ }^{9}$

Currently, RPLND performed in all patients with a residual mass $1 \mathrm{~cm}$ or greater is a de facto standard. ${ }^{7}$ Prediction models have been validated to be used to determine the need for post-chemotherapy RPLND. However, these have not been widely adopted. Specifically, we wonder if complexity, the patient subset used, ambiguity of terminology, and errors in reporting have contributed to lack of routine use of the Vergouwe nomogram.

Our study was quite consistent with published literature showing $60 \%$ of patients had malignancy and/or teratoma in pathology from RPLND. ${ }^{8}$ We identified and corrected

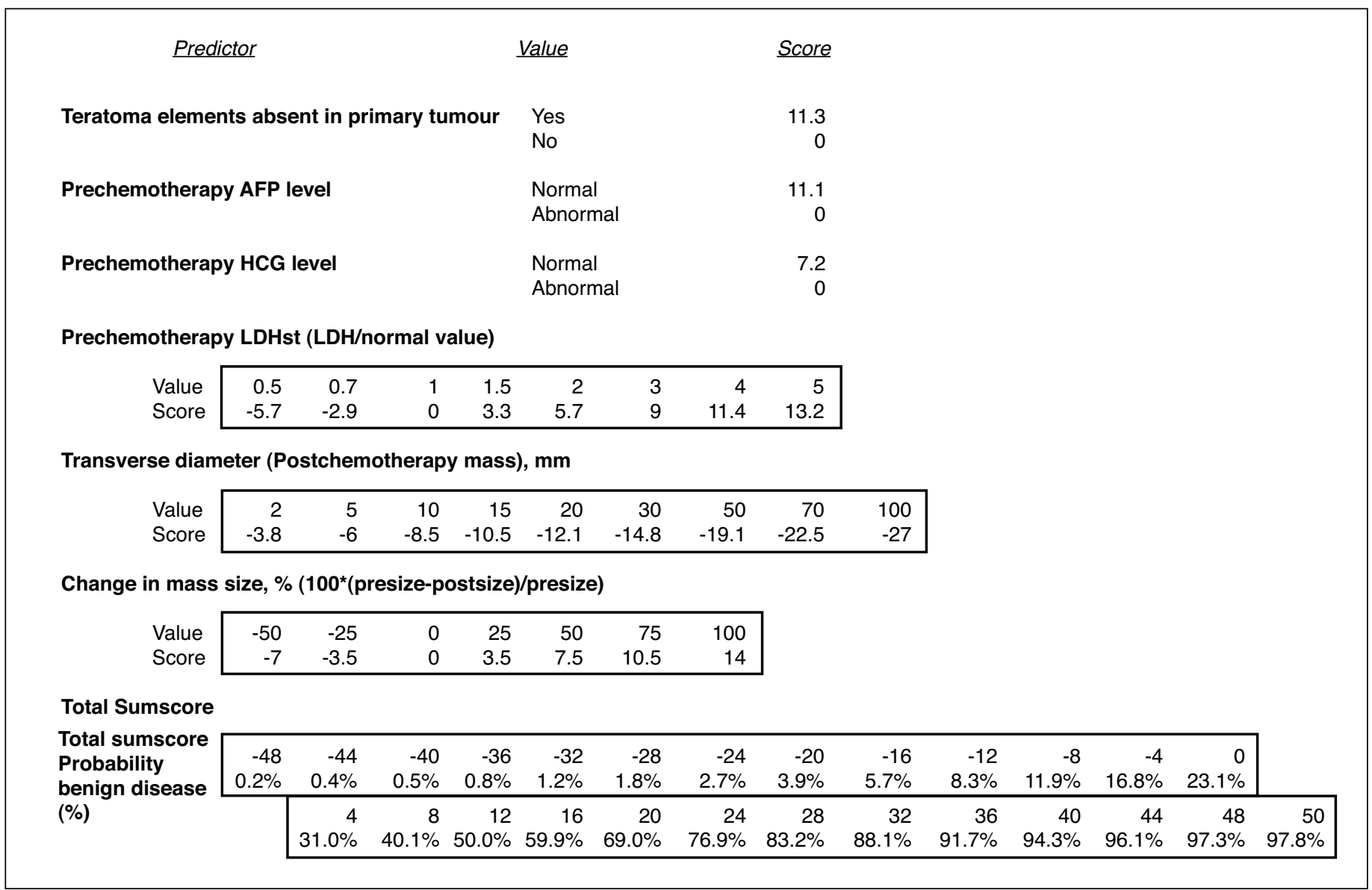

Fig. 3. Corrected nomogram. 
publication errors in a report of the largest validated nomogram based on more than 1000 patients at various academic centres worldwide, representing a very heterogeneous population. ${ }^{10}$ This model itself is not inherently flawed and incorporates many factors that have previously been used to predict the need for RPLND, including the presence of teratoma or mass shrinkage with chemotherapy, and is therefore comprehensive in its design. ${ }^{10}$ We applied the corrected version of this model with the suggested cutoff of greater than $70 \%$ probability of benign tissue; this cutoff allowed us to predict benign tissue in all those in whom it was predicted. Using such a cutoff may not interest all physicians, but it may be useful as an adjunct to make difficult decisions. ${ }^{10}$ We also generated an updated graphic nomogram for ease of use in clinical practice (Fig. 3).

Limitations of this study arise from the small sample size and its retrospective nature. A significant proportion of patients had incomplete data, which did not allow application of the predictive formula. We also did not include patients without RPLND in our study. Future collaborative studies with other large centres would provide more robust sample sizes. Comparison of patients without RPLND after five years would be an additional area of interest and future study. Similar to the methods of Vergouwe et al, we considered pathology "non-benign" in patients with both cancer and/or teratoma, but these two entities may develop differently. ${ }^{10,15}$ Radiological measures were based on reports rather than on re-measurement or central review, but that may be more reflective of actual clinical application. ${ }^{10}$ Our study also did not follow or predict outcomes for patients who did not initially undergo RPLND but did require further salvage treatment, and this may be another area for further study. Another inherent limitation is that the data for which the original nomogram was validated does refer to many large subspecialized cancer centres with possible variability in practice. This patient population is very heterogeneous and may not be representative of many treatment centres.

Once corrected for publication errors that made it difficult to understand and use, we found a previously validated nomogram to be an effective predictor in metastatic nonseminomatmous patients with post-chemotherapy residual masses. Many centres are cautious using this model, given the subset of patients for which it was created. We, however, validated this nomogram using data from a standard tertiary hospital and cancer centre that uses a $1 \mathrm{~cm}$ retroperitoneal lymphadenopathy cutoff for RPLND selection. Although the nomogram does require six clinical elements to be used and may be perceived as tedious, it can be simplified as a formula on an electronic spreadsheet for repeated use.

As current practice has shifted toward a more aggressive stance with RPLND, the corrected nomogram and this pre- dictive and clinically useful cutoff, pending further validation from other larger datasets, may aid in decision-making to avoid potentially unnecessary surgery.

Competing interests: Dr. Power has participated in clinical trials for Argos Therapeutics. Dr. Winquist has participated in clinical trials for AstraZeneca, Exelixis, Janssen, Medivation, and Roche. The remaining authors report no competing personal or financial interests.

This paper has been peer-reviewed.

\section{References}

1. Jemal A, Siegel R, Xu J, et al. Cancer statistics, 2010. CA Cancer J Clin 2010;60:277-300. http://dx.doi.org/10.3322/caac.20073

2. McGlynn KA, Devesa SS, Graubard Bl, et al. Increasing incidence of testicular germ cell tumours among black men in the United States. J Clin Oncol 2005;23:5757-61. http://dx.doi.org/10.1200/ JC0.2005.08.227

3. Powles JW, Zatonski W, Vander Hoorn S, et al The contribution of leading diseases and risk factors to excess losses of healthy life in Eastern Europe: Burden of disease study. BMC Public Health 2005;5:116. http://dx.doi.org/10.1186/1471-2458-5-116

4. Vergouwe $Y$, Steyerberg EW, Foster RS, et al. Validation of a prediction model and its predictors for the histology of residual masses in non-seminomatous testicular cancer. J Urol 2001;165:84-8; discussion 8. http://dx.doi.org/10.1097/00005392-200101000-00021

5. Spiess PE, Brown GA, Pisters LL, et al. Viable malignant germ cell tumour in the post-chemotherapy retroperitoneal lymph node dissection specimen: Can it be predicted using clinical parameters? Cancer 2006;107:1503-10. http://dx.doi.org/10.1002/cncr.22181

6. Kobayashi T, Kawakita M, Terachi T, et al. Significance of elevated preoperative alpha-fetoprotein in post-chemotherapy residual tumour resection for the disseminated germ cell tumours. I Surg Oncol 2006;94:619-23. http://dx.doi.org/10.1002/iso.20418

7. Riggs SB, Burgess EF, Gaston KE, et al. Post-chemotherapy surgery for germ cell tumours - what have we learned in 35 years? Oncologist 2014;19:498-506. http://dx.doi.org/10.1634/theoncologist.2013-0379

8. Steyerberg EW, Keizer HJ, Fossa SD, et al. Prediction of residual retroperitoneal mass histology after chemotherapy for metastatic non-seminomatous germ cell tumour: Multivariate analysis of individual patient data from six study groups. J Clin Oncol 1995;13:1177-87.

9. Vergouwe $Y$, Steyerberg EW, Eijkemans MJ, et al. Validity of prognostic models: When is a model clinically useful? Semin Urol Oncol 2002;20:96-107. http://dx.doi.org/10.1053/suro.2002.32521

10. Vergouwe Y, Steyerberg EW, Foster RS, et al. Predicting retroperitoneal histology in post-chemotherapy testicular germ cell cancer: A model update and multicentre validation with more than 1000 patients. Eur Urol 2007;51:424-32. http://dx.doi.org/10.1016/i.eururo.2006.06.047

11. Vergouwe $Y$, Steyerberg EW, de Wit R, et al. External validity of a prediction rule for residual mass histology in testicular cancer: An evaluation for good prognosis patients. Br I Cancer 2003;88:843-7. http://dx.doi.org/10.1038/si.bic.6600759

12. Newcombe RG. Two-sided confidence intervals for the single proportion: Comparison of seven methods. Stat Med 1998;17:857-72. http://dx.doi.org/10.1002/(SICI) 1097-0258(19980430) 17:8<857::AlDSIM777>3.0.C0;2-E

13. Streiner D, Norman G. PDQ Epidemiology 2nd ed. Toronto, ON: Mosby; 1996.

14. Albers $\mathrm{P}$, Weissbach L, Krege $\mathrm{S}$, et al. Prediction of necrosis after chemotherapy of advanced germ cell tumours: Results of a prospective multicentre trial of the German Testicular Cancer Study Group. I Urol 2004;171:1835-8. http://dx.doi.org/10.1097/01.ju.0000119121.36427.09

15. Carver BS, Bianco FJ Jr, Shayegan B, et al. Predicting teratoma in the retroperitoneum in men undergoing post-chemotherapy retroperitoneal lymph node dissection. J Urol 2006; 176:100-3; discussion 3-4. hittp:// dx.doi.org/10.1016/50022-5347(06)00508-8

Correspondence: Dr. Nahid Punjani, Division of Urology, Western University and London Health Sciences Centre, London, ON, Canada; nahidpunjani@gmail.com 